\title{
Neurophysiological correlates of tactile width discrimination in humans
}

Miguel Pais-Vieira ( $\nabla$ miguelpaisvieira@ua.pt)

Universidade de Aveiro

\section{Pais-Vieira Carla}

Universidade Católica Portuguesa

Allahdad Mehrab

Universidade Católica Portuguesa

Perrotta André

University of Coimbra (CISUC)

Peres André

University of Coimbra

Kunicki Carolina

Santos Dumont Institute

Oliveira Manuel

Universidade de Aveiro

\section{Research Article}

Keywords:

Posted Date: February 28th, 2022

DOI: https://doi.org/10.21203/rs.3.rs-1328335/v1

License: (c) (1) This work is licensed under a Creative Commons Attribution 4.0 International License.

Read Full License 


\section{Abstract}

Tactile information processing requires the integration of sensory, motor, and cognitive information. Width discrimination has been extensively studied in rodents, but not in humans. Here, we describe Electroencephalography (EEG) signals in humans performing a tactile width discrimination task. Comparison of changes in Spectral Power Density during two different periods of the task corresponding to the discrimination of the tactile stimulus and the motor response, revealed the engagement of a complex network associated with the electrodes recording from fronto-temporo-parieto-occipital areas and across multiple frequency bands. Analysis of ratios of higher [Ratio1:(0.5-20Hz)/(0.5-45Hz)] or lower frequencies [Ratio2: $(0.5-4.5 \mathrm{~Hz}) /(0.5-9 \mathrm{~Hz})$ ], also revealed that the periods of tactile discrimination and motor response, were accompanied by changes in these ratios. Further analysis of the tactile discrimination period demonstrated that ratios of frequencies recorded from electrodes located in frontoparietal regions were correlated to tactile width discrimination performance between subjects, while the dynamics in parietal and occipital electrodes were correlated to the changes in performance within subjects. These results support the notion that a bilateral asymmetrical widespread network is associated with tactile width discrimination.

\section{Highlights}

- Changes in multiple frequency bands occur during tactile width discrimination

- Activity from fronto-parietal electrodes correlates to performance

- Activity from parieto-occipital electrodes correlates to changes in performance

\section{Introduction}

Tactile discrimination processing requires the integration of sensory, motor, and cognitive information in humans and animals [1-8]. Width discrimination is a specific type of tactile information processing that has been extensively studied in rodents [2-4,9-12], but remains largely undescribed in humans" [13]. Width discrimination in the sub-centimeter scale is not likely to constitute a critical survival skill for humans, contrary to rodents $[14,15,16]$. Nonetheless, the large body of knowledge gathered in the latter species, can significantly inform us about the neural basis of somatosensory processing and lead to new lines of inquiry regarding this function in humans. For example, studies in rodents have revealed that width discrimination performance is associated with widespread and dynamic interactions involving information transfer in theta, beta, and gamma frequency bands in a fronto-parieto-occipital network [5].

To take advantage of the extensive body of knowledge existing in width discrimination in rodents, we have recently developed a width discrimination task for humans [13] that mimics the original rodent task [9]. In the version tested here (i.e., the passive version), subjects were required to insert the index finger in a small chamber and wait for the tactile stimulus to be delivered by two movable and non-visible bars (Fig. 1). In each trial, the bars moved towards the finger forming one of two widths: "Wide" or "Narrow". 
The subject was then required to make a motor response in one of two pushbuttons, to indicate which stimulus was delivered. As there is still very little research on this topic, it is not known if the same extensive network of regions and frequency bands involved in width discrimination in rodents is also present in humans. Preliminary data from a small number of subjects suggested that changes in the low gamma band across a network of electrodes recording from multiple regions may be relevant for tactile width discrimination [13].

Previous studies of EEG activity in other tactile discrimination paradigms indicate the engagement of a complex network of regions involving the somatosensory cortex, prefrontal cortex, and the parietal cortex, typically in alpha, mu, and beta frequency bands [17-24]. These studies have, mostly, explored EEG correlates of tactile processing using stimulation current, textures, or vibratory stimuli; due to their ability to generate EEG evoked responses. For example, previous studies have found that changes in the alpha frequency band in the $\mathrm{C} 3$ electrode are correlated to tactile performance [24]. However, based on our previous study [13], analysis of passive tactile width discrimination in humans may benefit from analysis of longer periods (e.g., larger than 1.0 second) due to the relatively large period of contact occurring between the bars and the finger. Comparison of ratios of frequency bands has been previously used to describe neurophysiological correlates and state dynamics when relatively large time intervals are involved, namely for cortical function in rodents [25-27]. This analysis compares two ratios, one with higher frequencies and another with lower frequencies, generating two different coordinates that can be used to identify states and transitions. Such analysis has previously allowed making inferences about the neurophysiological bases of ongoing natural behaviors [25] or broad neurophysiological states induced by pharmacological manipulation [27]. These previous studies, therefore support the use of these ratios of power to describe tactile width discrimination.

Here, we hypothesized that: $\mathrm{H} 1$ : EEG correlates of tactile width discrimination would change between the discrimination (i.e., tactile stimulus delivery) and the response (i.e., motor response) periods; $\mathrm{H} 2$ : that alpha band power in the $\mathrm{C} 3$ electrode during the discrimination period would predict tactile performance; H3: that state maps composed by ratios of different frequency bands would differentiate between these two periods; $\mathrm{H} 4$ : that these ratios would correlate to the behavioral performance; and lastly, H5: tactile width discrimination would be associated with changes in neural activity recorded from frontal, parietal, and occipital electrodes.

To describe the neurophysiological correlates of tactile width discrimination in humans we have recorded EEG activity from subjects performing a passive version of the task. First, the neurophysiological data recorded during the discrimination period was compared to the response period to describe the overall pattern of activity in these two different periods. Then, neural activity during the discrimination period was compared with tactile the behavioral performance using state maps composed by ratios of higher and lower frequencies.

\section{Results}


The present results include behavioral (17 sessions, in a total of 33 runs) and/or neurophysiological data (30 runs) from 17 subjects ( $9 / 17=52.94 \%$ female; with $28.9 \pm 7.28$ years old; min: 18 years old, max: 40 years old). The behavioral performance in the task was $76.55 \% \pm 14.32$ and the response latency was $1.045 \pm 0.266$ seconds. Response latency was not correlated to performance (Rs=0.1277, $P=0.5091$, $\mathrm{Q}=0.8265$ ). In the 17 sessions (each subject was schedule to perform two runs), a total of 33 runs were performed. Two subjects presented a performance below $50 \%$ in the first run and their analysis was not included in the results presented, except when specifically indicated. In four subjects, only data from one run is presented. Three of these runs were not analyzed due to the low quality of the recorded signals $(\mathrm{N}=3)$. The fourth run was from a subject who performed below $50 \%$ and, in addition, which had to be removed from the study due to excessive stress generated by the task. For the fourteen subjects that completed both runs, and overall improvement was found between the first and the second run (first run: $69.81 \pm 14.31 \%$ correct; second run: $85.36+-9.5 \%$; Paired samples $t$ test: $t=5.315$; $d f=13, P=0.0001$ ) (Fig. 1 d).

Analysis of power in delta $(0.5-4.5 \mathrm{~Hz})$, theta $(4.5-8.5 \mathrm{~Hz})$, alpha $(8.5-13.5 \mathrm{~Hz})$, beta $(13.5-30.5 \mathrm{~Hz})$, and low gamma $(30.0-45 \mathrm{~Hz})$ bands indicated that the periods of discrimination $(1500 \mathrm{~ms})$ (i.e., stimulus delivery) and response $(1500 \mathrm{~ms})$ (i.e., choosing and pressing one button) were characterized by fundamentally different patterns of activity throughout the network of electrodes recorded (Fig. $1 \mathrm{~d}$ ). Table 1 presents the statistical results for the comparison between the discrimination and response periods for each frequency band in each electrode. An overall reduction in power was present, frequently in more than one frequency band. The exception to this were electrodes F3, C4, and TP10; where no differences were found between the two periods analyzed for any frequency band. For channels with significant changes between the two periods, the theta frequency band was the most frequently affected $(10 / 13=76.9 \%$ channels), followed by alpha $(6 / 13=46.2 \%)$ and beta frequency bands $(5 / 13=38.5 \%)$. Lastly, delta $(4 / 13=30.8 \%)$ and low gamma bands $(3 / 13=23.1 \%)$ were less often affected. These results indicated that the discrimination and the response periods were associated, with a bilateral asymmetrical distribution of changes in power, occurring most commonly in theta, but also in other frequency bands in a network of electrodes recording from frontal, temporal, parietal, and occipital regions.

To test our second hypothesis, we compared the power in the alpha frequency band in the C3 electrode with tactile discrimination performance. No significant correlation was found between the performance and the power in the alpha frequency band for the discrimination period. (Rs $=0.007383, P=0.7035$, $\mathrm{Q}=0.8338$, n.s.).

To analyze changes occurring simultaneously in multiple frequency bands across the scalp, an analysis of state maps composed by ratios of frequency bands was performed [25] (Fig. 2 a-h). It has been previously shown that this analysis accurately captures global cortical dynamics and/or associated behaviors when multiple frequencies are involved [25,27]. As depicted in Fig. 2 panel a; ratio 1 (used to analyze changes occurring in higher frequency bands), was calculated as $(0.5-20 \mathrm{~Hz}) /(0.5-45 \mathrm{~Hz})$ and ratio2 (used to analyze changes occurring in lower frequency bands) was calculated as $(0.5-4.5 \mathrm{~Hz}) /(0.5-$ $9 \mathrm{~Hz}$ ). In Fig. 2 panel b, an example of the state maps for channel Fp2 during the discrimination (Dis) and 
response (Resp) periods is presented. The arrow tail corresponds to the ratio 1 and ratio 2 coordinates during the discrimination period, while the arrowhead corresponds to the ratio 1 and ratio 2 coordinates during the response period. The displacement of each vector reflects the neural dynamics occurring as the subject moved from a state associated with the discrimination to the neural state corresponding to response.

Analysis of these ratios revealed that the discrimination and response periods were characterized by different ratios of frequencies. In other words, the dynamics of change between the two behaviors corresponded to an overall increase in ratio 1 and ratio 2 throughout a network involving multiple electrodes (Table 2). Namely, significant differences were found for ratio 1 in all electrodes with the exception for Fp1, Fz, C4, Pz, and Tp10 (with Fp1, C4 and Tp10 presenting non-significant $\mathrm{P}$ values below 0.10). Meanwhile, for ratio 2 , significant differences were found for all electrodes, except for Fp1, F3, Cz, $\mathrm{C} 4,02$, and Tp10 (with Fp1 and Cz presenting $\mathrm{P}$ values below 0.10 ). A visual summary of these findings is depicted in Fig. $2 \mathrm{C}$, where the network of electrodes associated with significant differences in either or both ratios during the discrimination and response periods is presented.

Having determined that the discrimination and response periods were characterized by different ratios of higher and lower frequencies, we then analyzed if these differences could be relevant for the width discrimination task. More specifically, we asked if the variability observed in ratio1 and ratio 2 values during the discrimination period reflected the tactile performance in the task (note, for example, the large variability in the arrow tails depicted in Fig. 2 b). For this, ratio 1 and ratio 2 values were compared to the performances in each session using Spearman's Rho correlation (i.e., the value of the session was compared to the overall session ratio 1 and ratio 2 values). As presented in Fig. 2 (d), ratio 1 (left panel) and ratio 2 (right panel), significantly correlated to subjects' performance in a bilateral asymmetrical network across the scalp. For ratio 1, significant Spearman's Rho correlations were present in electrodes Fp2 (Rs=0.5545, $P=0.0018, Q=0.0192), F 4(R s=0.5285, P=0.0023, Q=0.02048) ; T 4(R s=0.5007, P=0.0057$, $\mathrm{Q}=0.026057), \mathrm{P} 3(\mathrm{Rs}=0.5796, \mathrm{P}=0.001, \mathrm{Q}=0.032), \mathrm{P} 4(\mathrm{Rs}=0.5647, \mathrm{P}=0.0014, \mathrm{Q}=0.0224)$, and $\mathrm{O} 2$ (Rs $=0.489, P=0.0071, Q=0.0284$ ). Meanwhile, for ratio 2 significant Spearman's Rho correlations were present for electrodes P3 (Rs=0.5416, $\mathrm{P}=0.0024, \mathrm{Q}=0.0192)$ and $\mathrm{P} 4(\mathrm{Rs}=0.5094, \mathrm{P}=0.0048, \mathrm{Q}=0.0256)$. These results indicated that the state maps composed by ratios of higher and lower frequencies allowed not only describing the dynamics associated with the two different periods of the task, but also, that these ratios reflected the overall performance in the task.

To determine if these correlations would still be present when task difficulty was changed, three subjects (that had already been previously tested in this task) performed one session with two runs in an easier version of the tactile task while EEG recordings were performed. For this the difference between the Wide and the Narrow stimuli was increased by $4.0 \mathrm{~mm}$. Under such conditions the behavioral performance was of $96.67 \pm 4.08 \%$. Examples of the state maps associated with these runs are shown in Fig. 2 panels $g-h$ (ratio $1 \mathrm{Fp} 2$ and ratio $2 \mathrm{P} 3$, respectively) where the red filled circles represent these additional runs. Comparison of the ratios of frequencies and the performance, when these six runs were pooled with the remaining sessions and analyzed, still generated significant correlations for channels Fp2 (Fig. 2 panel 
g), F4 and P4 in ratio 1 ( $F p 2: R s=0.4671, P=0.0047, Q=0.0376 ; F 4: R s=0.4422, P=0.0073, Q=0.0292 ; P 4$ : $R s=0.4232, P=0.0113 ; Q=0.0226$ ), but not for $T 4, P 3$, and 02 (T4: $R s=0.1202$, $P=0.4915, Q=0.4915$, n.s.; $P 3: R s=0.1711, P=0.3257, Q=0.3722$, n.s.; 02: $R s=0.3139, P=0.0663 ; Q=0.0884$, n.s.). Meanwhile, $a$ significant correlation between the performance and ratio 2 in electrode $P 3$ was maintained (P3: $R s=0.4457, P=0.0073, Q=0.0292$ ), but not for electrode $P 4$ (P4: Rs=0.3334, $P=0.00504, Q=0.08064$, n.s.). These results suggested that changing the degree of difficulty did not disrupt the overall correlation between neural activity and performance for channels Fp2, F4, P3 and P4.

As we have observed an improvement in performance between the first and the second runs that took place in each session (Fig. 1 c), and as we have observed significant correlations between the ratios of higher and lower frequencies in an asymmetrical network involving frontal, temporal, parietal, and occipital electrodes, we then asked if the dynamics of ratios in this network would reflect the dynamics of improvements in performance occurring between the first and the second run for the same subject. In other words, we asked to which extent these neurophysiological measures could reflect within-subjects differences in behavior. For this, we reanalyzed the data from the subsample of subjects that completed both runs ( $\mathrm{N}=14$ subjects in 28 runs; the subject that presented a performance below $50 \%$ in the first run and then improved in the second run was included in this analysis). First, we calculated the changes occurring in ratio 1 and ratio 2 in the same subject. In Fig. $3 a$ and b, the changes occurring in ratio 1 and ratio 2 of electrodes P4 (panel a) and 02 (panel b), between the first and the second runs of the session are presented. Then, we analyzed both ratios simultaneously, to determine if a clear pattern could be identified. In Fig. 3 panels $c$ and d, both coordinates are arranged to form the arrow tail (Ratio 1: X-axis, and Ratio 2: Y-axis in the first run) and the arrowhead (Ratio 1: X-axis, and Ratio 2: Y-axis in the second run) of a vector. While no clear pattern could be observed in the P4 electrode (Fig. $3 \mathrm{C}$ ), an overall shift towards the right lower quadrant could be identified in 02 (Fig. $3 \mathrm{~d}$ ). Although this suggested that changes occurring in ratios could be related to the differences between the first and the second run, no clear pattern could be identified. Then, the difference between neural activity in the two runs was calculated and plotted against the difference in performance. This allowed comparing the physiological and behavioral evolution throughout the session. In Fig. 3, panels e and f, show the difference in ratio 1 for the two runs simultaneously with the difference in performance. Even though no clear correlation could be observed between these two variables, visual inspection suggested that an overall increase in the variance of ratio 1 (i.e., increase in the variance of values in the coordinates axis) was present, as the difference in performance increased (i.e., abscissa values increased). To be able to explain this variation, we then tested if the symmetrical pattern observed in 02 (Fig. $3 \mathrm{f}$ ) converted to its absolute values (which now reflected "a change in performance" instead of "an improvement in performance") could explain the large variability in the data. As presented in Fig. 3 panels $\mathrm{g}$ and $\mathrm{h}$, a significant correlation was found between the dynamics of ratio 1 for channels P4 (P4 Ratio 1: Rs=0.7209, P=0.0036, Q $=0.0252$; note that this result is also significant if the same analysis is performed without subject 14) and 02 (02 Ratio 1: $R s=0.6452, P=0.0127, Q=0.04445$ ), but not for $F p 2$ (Ratio 1: Rs=0.1424, $P=0.6273, Q=0.73185$, n.s.), $F 4$ (F4 Ratio 1: $R s=-0.02225, P=0.9398, Q=0.9398$, n.s.), $T 4$ (T4 Ratio 2: $R s=0.3471, P=0.2241, Q=0.5229$, 
n.s.), P3 (P3 Ratio 2: Rs= $-0.1891, P=0.5173$, n.s.) or $P 4$ (P4 Ratio 2: $R s=0.2937, P=0.3082, Q=53935$, n.s.).

To further test if these dynamics were independent of the level of difficulty in the task, the six runs from the sessions of the small sample of three subjects presented in Fig. $2 \mathrm{~g}$ and $\mathrm{h}$, was combined with the remaining subjects and the pooled data was reanalyzed solely for channels $\mathrm{P} 4$ and $\mathrm{O} 2$. As presented in Fig. 3, panels $\mathrm{g}$ and $\mathrm{h}$, the inclusion of these additional runs still presented a significant correlation for $\mathrm{P} 4$ (Rs All $=0.6395, P=0.0057$ ) and for 02 (Rs All $=0.5541, P=0.0210$ ). These results indicated that the dynamics of ratio 1 for electrodes $\mathrm{P} 4$ and 02 encoded the changes in performance in the behavioral task between the two different runs of the same session.

\section{Discussion}

The main objective of this study was to describe EEG activity during tactile width discrimination in humans. Neural activity in the period of tactile stimulation and the period of response was characterized by multiple power changes across frequency bands and electrodes recorded, as well as in state maps composed of ratios of higher and lower frequencies. Detailed analysis of the discrimination period revealed that a network of electrodes recording from frontal, temporal, parietal, and occipital regions was significantly correlated to behavioral performances. When task difficulty was reduced and the pooled data was analyzed (i.e., regular and easy sessions), only fronto-parietal electrodes remained correlated to performances. Lastly, state map dynamics of ipsilateral occipito-parietal electrodes encoded the changes in performance within the same subject (i.e., between runs) independently of task difficulty. A summary of these findings is presented in Table 3.

Neural activity during the discrimination and response periods was characterized by an overall reduction in power occurring more often in theta, alpha, and beta frequency bands (but also in delta and low gamma bands), in a network that included Fp1-2-Fz, F4, T3-4, C3, P3-4-z, and 01-2 electrodes (only in electrodes F3, C4 and Tp10 no significant differences were found). These results support our first hypothesis that discrimination and response periods would be associated with power changes for specific frequency bands.

Power changes in theta, alpha, and beta frequency bands for the $\mathrm{C} 3$ electrode during discrimination and response periods, as well as the changes in ratios of frequencies, are in line with previous studies of tactile function. Tactile discrimination is often associated with changes in EEG activity recorded from the electrode $\mathrm{C} 3 / 4$, namely with the appearance of event related potentials or power changes in alpha and beta frequency bands [17-24,28-29]. For example, a relevant contribution of $\mathrm{mu}(8-15 \mathrm{~Hz})$ and beta (16$30 \mathrm{~Hz}$ ) frequency bands has been demonstrated for texture discrimination using a support vector machine classifier in electrodes recording from primary and secondary somatosensory cortex [23]. In addition, other complex functions such as orienting attention to an upcoming tactile event have also been associated with alpha and beta band modulation in sensorimotor regions [30]. Lastly, the theta frequency band in the parietal region has been reported to participate in the regulation of attention and emotion 
during tactile processing [31]. Gamma band oscillations in the primary somatosensory cortex have been associated with tactile processing in other tactile discrimination tasks [32,33], tactile spatial attention (namely, when simultaneous alpha and beta band suppression occurs in the parieto-occipital cortex) [34], and multisensory communication [35]. Here, no differences or significant changes in power were observed in the gamma frequency band for the $\mathrm{C} 3 / 4$ electrodes, possibly due to the long duration of the periods analyzed [13]. Altogether, the study of differences in power, indicates that multiple changes occurred for different frequency bands and electrodes during the discrimination and the response periods, and that power changes in the theta, alpha, and beta frequency bands for the $\mathrm{C} 3$ electrode were largely in line with previous reports.

\section{Ratios of frequencies predict performance}

Analysis of ratios of frequencies revealed that the transition between the discrimination and the response periods was characterized by an increase in both ratios occurring in electrodes Fp2, F4, T3-4, C3, P3-4, and 01 ; an increase in ratio 1 for $\mathrm{F} 3, \mathrm{Cz}$, and 02 electrodes; and an increase in ratio 2 for electrode $\mathrm{Pz}$. These results support our third hypothesis that the analysis of ratios of frequencies distinguished between the periods of discrimination and response. Ratios allowed identifying electrodes Fp2 and T4 as being associated with the behavioral performance, namely through changes in beta and gamma frequency bands (i.e., ratio 1). This occurred, even though no significant difference in power between discrimination and response periods was present in these specific frequency bands (Table 1).

An association between neural activity during the discrimination period and tactile discrimination acuity was found in a bilateral asymmetric network of electrodes spread throughout the scalp (Ratio 1 for electrodes Fp2, F4, T4, P3-4, and 02; and with Ratio 2 for electrodes P3-4). These results support our last two hypotheses concerning the ability to predict performance from neural activity analyzed with state maps ( $\mathrm{H} 4)$, and concerning the involvement of electrodes positioned in frontal, parietal, and occipital regions $(\mathrm{H} 5)$.

These electrodes were correlated with tactile performance mostly in ratio 1 which includes beta and gamma band frequencies. Tactile spatial attention has been associated with increases in gamma band $(60-95 \mathrm{~Hz})$ and decreases in alpha and beta bands [34] which could potentially help explain our findings (even though we have only analyzed low gamma frequency band).

\section{Occipito-parietal electrodes}

It has been proposed that simultaneous alpha, beta, and gamma frequency band oscillations are required for unified cognitive operations [35] such as attention to tactile stimuli [34], and attention to affective touch [31]. The large number of modulations observed across the different frequency bands and electrodes in our study is in line with this previous proposal. For example, decreases in the power of theta and beta frequency in the parietal regions during tactile information processing have been associated 
with attentional and emotional mechanisms [31], and alpha rhythm, originating from the occipito-parietal areas, is known to be involved in perception, especially in visual attention [37]. Our results are in line with these previous findings since the activity recorded from electrodes placed in occipito-parietal areas (P3, $P 4,01)$ presented differences in theta and alpha, in beta $(P 4,01)$, as well as in gamma (P3) frequency bands. Also, the activity from electrodes $\mathrm{P} 3, \mathrm{P} 4$, and $\mathrm{O} 2$, was correlated to the performance, and the activity in $\mathrm{P} 4$ and $\mathrm{O} 2$ was correlated to the overall change in performance.

The dynamics of ratio 1 in electrodes $\mathrm{P} 4$ and 02 predicted the amount of change in behavioral performance for the different runs of a given subject. These dynamics, however, did not separate improvements from impairments in performance (i.e., did not predict the direction of change). Therefore, this simultaneous change may reflect a construct that is related to performance, but not necessarily similar. It has been previously argued that task difficulty may be related to attention, engagement, arousal as well as other constructs or properties [38-42], and that lateralized activity in alpha frequency band recorded from sensorimotor regions may be a relevant neurophysiological feature of difficulty levels [42]. Our additional experiments with a small sample of subjects performing an easier version of the task, suggested that the neural correlates of these dynamics were independent of task difficulty. In future studies it will be important to clarify the relation between this neurophysiological correlate and the different cognitive aspects of width discrimination.

\section{Comparison to rodents}

The networks of electrodes and the frequency bands associated with significant activity in the width discrimination task frequently matched previous human or rodent studies. Therefore, the findings of the present study support the notion that a systematic description of tactile width discrimination in humans can significantly improve our current knowledge on tactile processing and benefit from the large body of knowledge existing in rodents [2-5,9-12]. In future studies it will be relevant to perform additional analysis including not only the different task periods and the individual subjects' performance, but also coherence and information transfer between the different channels and frequencies analyzed [4-5,12].

\section{Caveats, potential bias, and technical discussion}

A small number of caveats and potential bias should be considered. The number of electrodes used to record in this study does not allow for source analysis and therefore, the present findings are discussed regarding the position of each particular electrode rather than the cortical region beneath it. Analysis of ratio 1 in this study included low $(30.5-45.0 \mathrm{~Hz})$ but not the high gamma band frequencies which are relevant for tactile spatial attention [34]. We have opted for this because we have previously seen that these specific ratios seem to capture well the dynamics of tactile information processing in rodents, especially when analyzing longer periods [27]. Therefore, the present results and conclusions can be, to some extent, biased by this approach. 
Lastly, for one of our subjects, performing the task proved to be a stressful event. Such an effect of the task was not predictable from our previous [13] or current experience (now including more than 30 subjects tested in different versions of the task), since subjects are always allowed to interact with the task before the session (i.e., they perform a small number of trials before the actual session begins until they indicate being comfortable with the procedure). Even though, in future studies, we propose implementing a short psychological evaluation to subjects before testing them in this apparatus.

\section{Conclusion}

Tactile width discrimination was associated with changes in neural activity in networks involving electrodes from fronto-temporo-parieto-occipital networks, mostly in theta, alpha, and beta frequency bands. A bilateral asymmetrical network associated with ipsilateral fronto-temporo-parieto-occipital electrodes and contralateral parietal electrodes encoded tactile width discrimination performance. Signals recorded from fronto-parietal electrodes predicted tactile performance between subjects independently of task difficulty, while signals from parieto-occipital electrodes predicted changes in performance within subjects, independently of task difficulty. These results support and expand previous findings in rodents and humans on the widespread asymmetrical involvement of cortical networks during tactile width discrimination.

\section{Material And Methods}

\section{Subjects}

The present study was approved by the Ethics Committee of the University of Minho (SECVS 148/2016; and the Comité para as Ciências da Saúde of the Catholic University of Portugal (39/2017), according to the Code of Ethics of the World Medical Association (Declaration of Helsinki) for experiments involving humans. all experiments were performed in accordance with relevant named guidelines and regulations. All participating subjects voluntarily filled an informed consent. Three subjects were tested at the University of Minho (additional experiments performed with the easier version of the task), the remaining subjects were tested at the Catholic University of Portugal (subjects tested in the regular version of the task).

\section{Task}

The differential width discrimination task and the protocol used for the experiments described here have been previously described in detail elsewhere [13]. Briefly, in each trial, subjects were required to insert the right index finger in an invisible aperture formed between to movable bars ("Narrow" or "Wide") hidden behind the front panel (Fig. 1) and then to push one of two buttons to indicate the corresponding width. The sequence of actions to be performed by the subjects was indicated by different lights in the front panel. 
In each session subjects performed a passive version of the task (as previously described in more detail [13]) with two runs. In the passive version of the task subjects inserted their index finger in the box and the bars moved towards the finger. In each trial the subject was required to wait for the bars to move and form the aperture width. After a period of $1000 \mathrm{~ms}$ they would automatically return to their initial position and the subject had to make a behavioral response in one of two push buttons to indicate the width. A total of 20 Wide and 20 Narrow trials were performed in each run of the task, in a total of 80 trials per session. Except for the three subjects who were tested in the easier version of the task, all other subjects performed only in one session.

\section{EEG recordings}

EEG recordings were made 16 channels using a 10-20 placement (V-Amp, actiCAP; Brain Products GmbH, Gilching, Germany). Signals were recorded using the Brain Vision Recorder (version 2.1.0, Brain Products, Gilching, Germany) and analyzed using Brain Vision Analyzer (version 2.2.1, Brain Products, Gilching, Germany) and Matlab (Mathworks, 2018b, Natick, USA). Pre-processing included re-referencing using all channels as reference [43]. Then a notch filter was applied $(50 \mathrm{~Hz})$. Ocular correction was performed using the Gratton and Coles algorithm (already implemented in Visual Analyzer). The data was then segmented according to the discrimination and response marker, with a window of $1500 \mathrm{~ms}$ (-500 up to $1000 \mathrm{~ms}$ after each marker), ensuring no overlap between the two actions. Fast Fourier Transform with a resolution of $0.5 \mathrm{~Hz}$ was then applied. Frequency bands were analyzed as: delta $(0.5-4.5 \mathrm{~Hz})$, theta $(4.5-8.5 \mathrm{~Hz})$, alpha $(8.5-13.5 \mathrm{~Hz})$, beta $(13.5-30.5 \mathrm{~Hz})$, and low gamma $(30.5-45 \mathrm{~Hz})$. Data was only analyzed up to $45 \mathrm{~Hz}$ (described here as low gamma frequency band), to match the state map values used in a previous study [27]. Power was then normalized across the different subjects and compared for the different periods (discrimination and response). Comparison of power was made between the two periods, instead of using a baseline, because we have observed in a previous study that large intertrial intervals quickly led to mental exhaustion of participants. Also, as the moving bars require a relatively long period of time to stimulate the subject, we have opted to analyze long periods of time. Altogether, for this version of the task, we have opted to reduce the intertrial interval, and compare the neural signals between the discrimination and the reward periods.

\section{Ratios of frequencies analysis}

Analysis of state maps was based on an adaptation [27] of the original method described by Gervasoni and colleagues [25]. This method calculates two different ratios (ratio 1 and ratio 2) based on the average power found in higher and lower frequencies, namely: ratio1, R1:(0.5-20Hz)/(0.5-45Hz)] and ratio 2, R2: $(0.5-4.5 \mathrm{~Hz}) /(0.5-9 \mathrm{~Hz})$. Each of these ratios was then be used as a coordinate (Ratio 1: abscissa, Ratio 2: ordinate) in a state map.

\section{Statistical analysis}

Results are presented as Mean and standard deviation (Mean \pm SD). Paired samples t tests or Wilcoxon signed ranks tests were used to compare power in each frequency band between the two different periods and to compare within-subjects neurophysiological changes (i.e., between the first and the second run). 
Two-way repeated measures (Period X Ratio) ANOVA was used to compare the two ratios in the two different periods. Spearman's Rho was used to correlate subjects' performances with the Power Ratios. This analysis was followed by Benjamini and Hochbergh's correction for false discovery rate [43]. The $Q$ values indicating the corrected statistic are presented in the text immediately after the original $P$ values. Significance was considered for alpha at $5 \%$ with the exceptions where multiple comparisons were performed.

\section{Declarations}

\section{Acknowledgments:}

BIAL Foundation 95/2016 (MPV, CPV, MA). This work was financially supported by National Funds through FCT - Fundação para a Ciência e a Tecnologia, I.P., under the projects UIDP/04501/2020 (MPV), UIDB/04279/2020 (CPV), FCT/IF/00098/2015 (MPV), project CISUC - UID/CEC/00326/2020 (AP) and by European Social Fund, through the Regional Operational Program Centro 2020 (AP).

\section{Author contributions:}

$\mathrm{CPV}, \mathrm{AM}, \mathrm{AP}$, and MPV collected the data; CPV, OM, CK, PA, and MPV analyzed the data; all authors participated in the manuscript writing and agreed to the final version of the manuscript.

\section{Data availability:}

The datasets generated and/or analysed during the current study are available in the Open Science Frame repository in the following link:

https://osf.io/sbnqe/?view_only=6634716552c74f5aa6d1cf64d693fd10

\section{Competing interests statement:}

The authors declare no competing financial and non-financial interests.

\section{References}

1. Chapman, C.E. and Ageranioti-Bélanger, S.A. Discharge properties of neurones in the hand area of primary somatosensory cortex in monkeys in relation to the performance of an active tactile discrimination task. Experimental brain research, 87(2), pp.319-339 (1991).

2. Krupa, D.J., Wiest, M.C., Shuler, M.G., Laubach, M. and Nicolelis, M.A. Layer-specific somatosensory cortical activation during active tactile discrimination. Science, 304(5679), pp.1989-1992 (2004). 
3. Pais-Vieira, M., Lebedev, M.A., Wiest, M.C. and Nicolelis, M.A. Simultaneous top-down modulation of the primary somatosensory cortex and thalamic nuclei during active tactile discrimination. Journal of Neuroscience, 33(9), pp.4076-4093 (2013a).

4. Pais-Vieira, M., Kunicki, C., Tseng, P.H., Martin, J., Lebedev, M. and Nicolelis, M.A. Cortical and thalamic contributions to response dynamics across layers of the primary somatosensory cortex during tactile discrimination. Journal of neurophysiology, 114(3), pp.1652-1676 (2015).

5. Kunicki, C., C Moioli, R., Pais-Vieira, M., Salles Cunha Peres, A., Morya, E. and AL Nicolelis, M. Frequency-specific coupling in fronto-parieto-occipital cortical circuits underlie active tactile discrimination. Scientific reports, 9(1), pp.1-14 (2019).

6. O'Doherty, J.E., Lebedev, M.A., Ifft, P.J., Zhuang, K.Z., Shokur, S., Bleuler, H. and Nicolelis, M.A. Active tactile exploration using a brain-machine-brain interface. Nature, 479(7372), pp.228-231 (2011).

7. Simões-Franklin, C., Whitaker, T.A. and Newell, F.N. Active and passive touch differentially activate somatosensory cortex in texture perception. Human brain mapping, 32(7), pp.1067-1080 (2011).

8. Adhikari, B.M., Sathian, K., Epstein, C.M., Lamichhane, B. and Dhamala, M. Oscillatory activity in neocortical networks during tactile discrimination near the limit of spatial acuity. Neuroimage, 91, pp.300-310 (2014).

9. Krupa, D.J., Matell, M.S., Brisben, A.J., Oliveira, L.M. and Nicolelis, M.A. Behavioral properties of the trigeminal somatosensory system in rats performing whisker-dependent tactile discriminations. Journal of Neuroscience, 21(15), pp.5752-5763 (2001).

10. Wiest, M.C., Thomson, E., Pantoja, J. and Nicolelis, M.A. Changes in S1 neural responses during tactile discrimination learning. Journal of neurophysiology, 104(1), pp.300-312 (2010).

11. Pais-Vieira, M., Lebedev, M., Kunicki, C., Wang, J. and Nicolelis, M.A. A brain-to-brain interface for realtime sharing of sensorimotor information. Scientific reports, 3(1), pp.1-10 (2013b).

12. Thomson, E., Lou, J., Sylvester, K., McDonough, A., Tica, S. and Nicolelis, M.A. Basal forebrain dynamics during a tactile discrimination task. Journal of neurophysiology, 112(5), pp.1179-1191 (2014).

13. Perrotta, A., Pais-Vieira, C., Allahdad, M.K., Bicho, E. and Pais-Vieira, M. Differential width discrimination task for active and passive tactile discrimination in humans. Methods $X, 7$, p.100852 (2020).

14. Vincent S.B. The function of the vibrissae in the behavior of the white rat. Behav Monographs 1:1-82 (1912).

15. Carvell GE and Simons D.J. Biometric analyses of vibrissal tactile discrimination in the rat. $J$ Neurosci 10:2638-2648 (1990).

16. Brecht, M., Preilowski, B. and Merzenich, M.M. Functional architecture of the mystacial vibrissae. Behavioural brain research, 84(1-2), pp.81-97 (1997).

17. Pfurtscheller, G., Woertz, M., Krausz, G. and Neuper, C. Distinction of different fingers by the frequency of stimulus induced beta oscillations in the human EEG. Neuroscience letters, 307(1), pp.49-52 (2001). 
18. Spitzer, B. and Blankenburg, F. Stimulus-dependent EEG activity reflects internal updating of tactile working memory in humans. Proceedings of the National Academy of Sciences, 108(20), pp.84448449 (2011).

19. Ishigaki, T., Ueta, K., Imai, R. and Morioka, S. EEG frequency analysis of cortical brain activities induced by effect of light touch. Experimental brain research, 234(6), pp.1429-1440 (2016).

20. Moungou, A., Thonnard, J.L. and Mouraux, A. EEG frequency tagging to explore the cortical activity related to the tactile exploration of natural textures. Scientific reports, 6(1), pp.1-9 (2016).

21. Genna, C., Oddo, C.M., Fanciullacci, C., Chisari, C., Jörntell, H., Artoni, F. and Micera, S. Spatiotemporal dynamics of the cortical responses induced by a prolonged tactile stimulation of the human fingertips. Brain topography, 30(4), pp.473-485 (2017).

22. Whitmarsh, S., Oostenveld, R., Almeida, R. and Lundqvist, D. Metacognition of attention during tactile discrimination. Neurolmage, 147, pp.121-129 (2017).

23. Eldeeb, S., Weber, D., Ting, J., Demir, A., Erdogmus, D. and Akcakaya, M. EEG-based trial-by-trial texture classification during active touch. Scientific reports, 10(1), pp.1-13 (2020).

24. Su, S., Chai, G., Sheng, X., Meng, J. and Zhu, X. Contra-lateral desynchronized alpha oscillations linearly correlate with discrimination performance of tactile acuity. Journal of Neural Engineering, $17(4)$, p.046041 (2020).

25. Gervasoni, D., Lin, S.C., Ribeiro, S., Soares, E.S., Pantoja, J. and Nicolelis, M.A. Global forebrain dynamics predict rat behavioral states and their transitions. Journal of Neuroscience, 24(49), pp.11137-11147 (2004).

26. Pereira, A., Ribeiro, S., Wiest, M., Moore, L.C., Pantoja, J., Lin, S.C. and Nicolelis, M.A. Processing of tactile information by the hippocampus. Proceedings of the National Academy of Sciences, 104(46), pp.18286-18291 (2007).

27. Pais-Vieira, M., Kunicki, C., Peres, A. and Sousa, N. Ceftriaxone modulates the acute corticosterone effects in local field potentials in the primary somatosensory cortex of anesthetized mice. Scientific reports, 9(1), pp.1-11 (2019).

28. Baumgarten, T.J., Schnitzler, A. and Lange, J. Beta oscillations define discrete perceptual cycles in the somatosensory domain. Proceedings of the National Academy of Sciences, 112(39), pp.1218712192 (2015).

29. Baumgarten, T.J., Schnitzler, A. and Lange, J. Prestimulus alpha power influences tactile temporal perceptual discrimination and confidence in decisions. Cerebral Cortex, 26(3), pp.891-903 (2016).

30. Van Ede, F., De Lange, F., Jensen, O. and Maris, E. Orienting attention to an upcoming tactile event involves a spatially and temporally specific modulation of sensorimotor alpha-and beta-band oscillations. Journal of Neuroscience, 31(6), pp.2016-2024 (2011).

31. von Mohr, M., Crowley, M.J., Walthall, J., Mayes, L.C., Pelphrey, K.A. and Rutherford, H.J. EEG captures affective touch: CT-optimal touch and neural oscillations. Cognitive, Affective, \& Behavioral Neuroscience, 18(1), pp.155-166 (2018). 
32. Michail, G., Dresel, C., Witkovský, V., Stankewitz, A. and Schulz E. Neuronal oscillations in various frequency bands differ between pain and touch. Frontiers in human neuroscience, 10, p.182 (2016).

33. Jiao, J., Hu, X., Huang, Y., Hu, J., Hsing, C., Lai, Z., Wong, C. and Xin, J.H. Neuro-perceptive discrimination on fabric tactile stimulation by Electroencephalographic (EEG) spectra. Plos one, 15(10), p.e0241378 (2020).

34. Bauer, M., Oostenveld, R., Peeters, M. and Fries, P. Tactile spatial attention enhances gamma-band activity in somatosensory cortex and reduces low-frequency activity in parieto-occipital areas. Journal of Neuroscience, 26(2), pp.490-501 (2006).

35. Misselhorn, J., Schwab, B.C., Schneider, T.R. and Engel, A.K. Synchronization of sensory gamma oscillations promotes multisensory communication. Eneuro, 6(5) (2019.

36. Palva, S. and Palva, J.M. New vistas for a-frequency band oscillations. Trends in neurosciences, 30(4), pp.150-158 (2007).

37. Romei, V., Gross, J. and Thut, G. On the role of prestimulus alpha rhythms over occipito-parietal areas in visual input regulation: correlation or causation?. Journal of Neuroscience, 30(25), pp.8692-8697 (2010).

38. Pope, A.T., Bogart, E.H. and Bartolome, D.S. Biocybernetic system evaluates indices of operator engagement in automated task. Biological psychology, 4O(1-2), pp.187-195 (1995).

39. Brouwer, A.M., Hogervorst, M.A., Van Erp, J.B., Heffelaar, T., Zimmerman, P.H. and Oostenveld, R. Estimating workload using EEG spectral power and ERPs in the n-back task. Journal of neural engineering, 9(4), p.045008 (2012).

40. Heard, J., Harriott, C.E. and Adams, J.A. A survey of workload assessment algorithms. IEEE Transactions on Human-Machine Systems, 48(5), pp.434-451 (2018).

41. Faller, J., Cummings, J., Saproo, S. and Sajda, P. Regulation of arousal via online neurofeedback improves human performance in a demanding sensory-motor task. Proceedings of the National Academy of Sciences, 116(13), pp.6482-6490 (2019).

42. Jao, P.K., Chavarriaga, R., Dell'Agnola, F., Arza, A., Atienza, D. and Millán, J.D.R. EEG correlates of difficulty levels in dynamical transitions of simulated flying and mapping tasks. IEEE Transactions on Human-Machine Systems, 51(2), pp.99-108 (2020).

43. Bertrand, O., Perrin, F. and Pernier, J. A theoretical justification of the average reference in topographic evoked potential studies. Electroencephalography and Clinical Neurophysiology/Evoked Potentials Section, 62(6), pp.462-464 (1985).

44. Benjamini, Y. and Hochberg, Y. Controlling the false discovery rate: a practical and powerful approach to multiple testing. Journal of the Royal statistical society: series B (Methodological), 57(1), pp.289300 (1995).

\section{Tables}

Tables are only available as a download in the Supplemental Files section. 


\section{Figures}
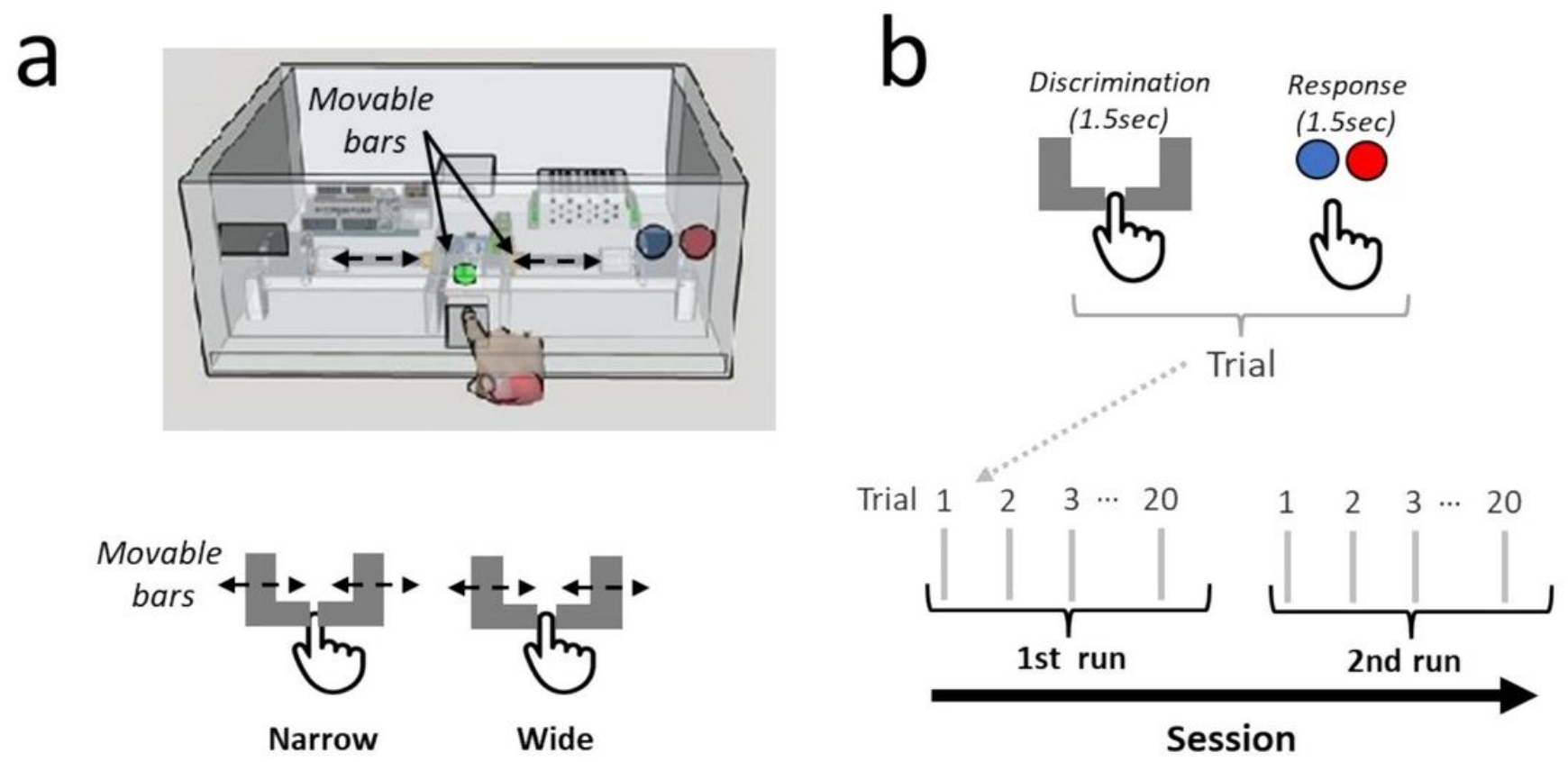

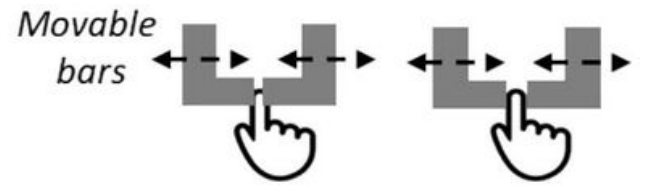

Narrow

Stimulus

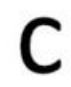

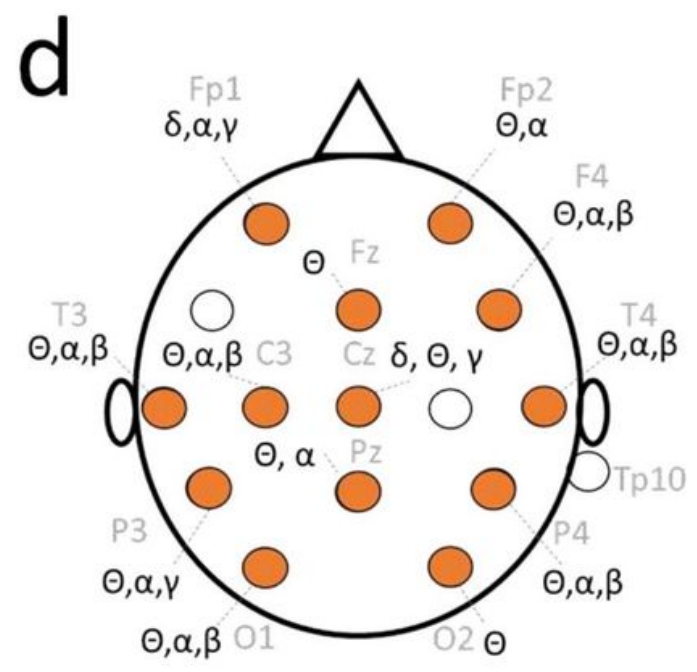

Session

Figure 1

Study design, behavioral performance, and differences in power of frequency bands. a) Tactile width discrimination task and representation of narrow and wide stimuli delivered to the index finger of the subject (i.e., passive tactile stimulation). In both Wide and Narrow stimuli, the bars touched the index finger. b) In each trial, the discrimination period and the response period were analyzed. Sessions consisted of two runs of 20 trials, each with 10 trials with the narrow stimulus and 10 trials with the wide stimulus. c) An overall improvement in performance was observed between the first run (empty or red circles) and the second run (black filled circles). Red filled circles indicate two subjects that presented 
performances below chance. d) Comparison of power in the main frequency bands revealed an extensive network of electrodes with significant differences (orange circles) between the discrimination and the response periods (also see text and Table 1 for details). Note that for the gamma frequency band only the lower part of the spectra was analyzed (low gamma: 30.0-45.0 Hz).

a

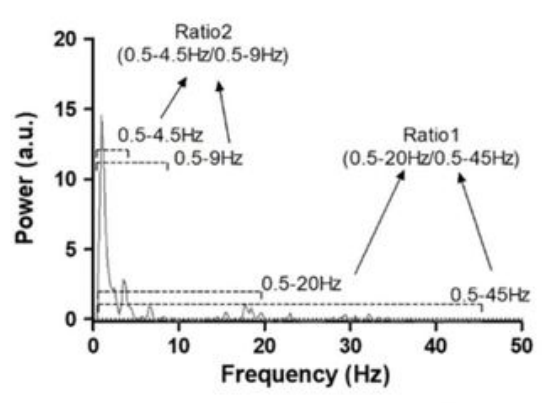

b

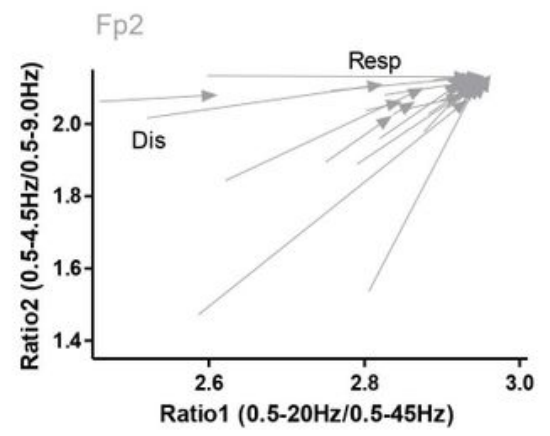

C

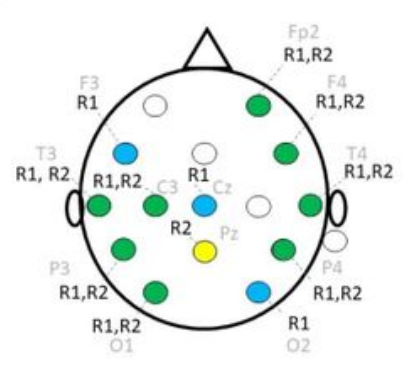

e

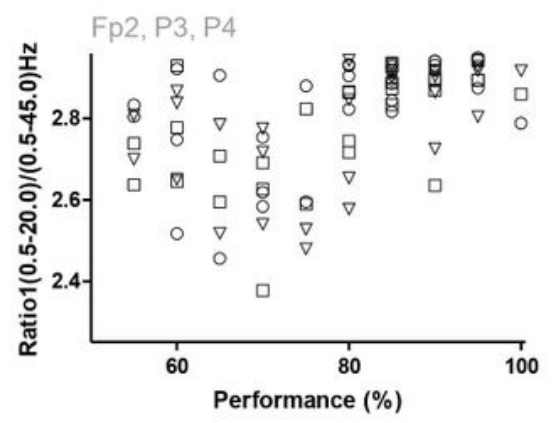

g

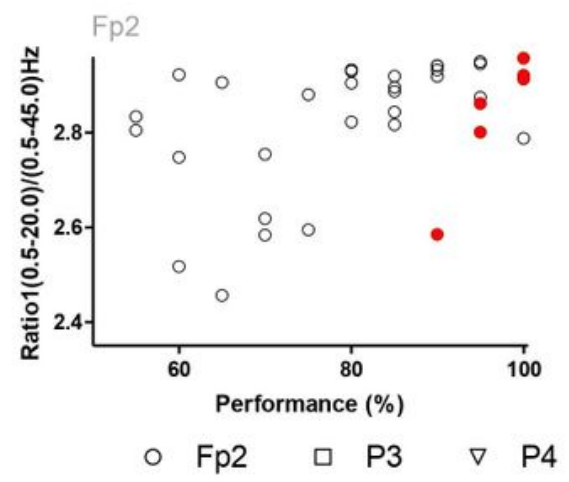

d
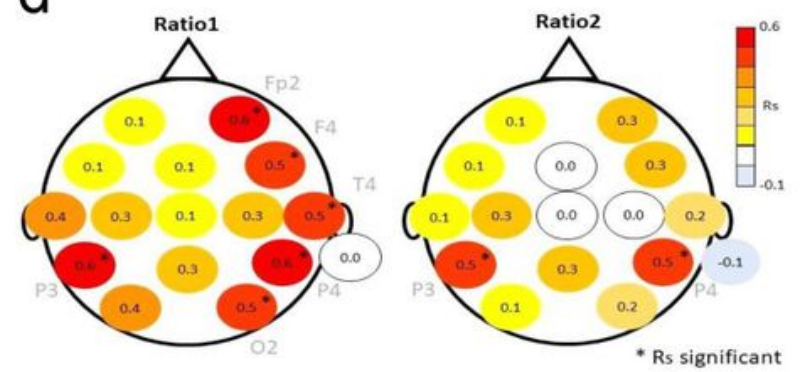

f

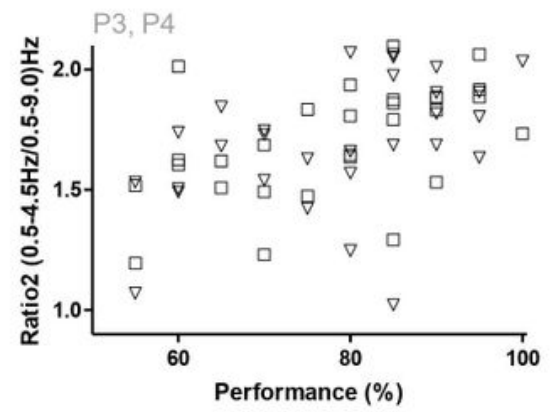

$\mathrm{h}$

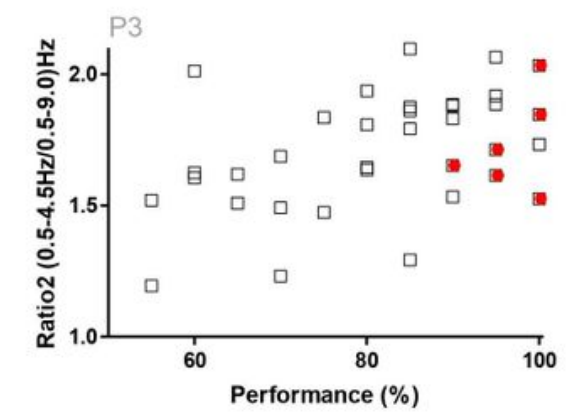

Fp2 easy

- P3 easy 


\section{Figure 2}

Ratios of frequencies are correlated to tactile performance. a) State maps were calculated using two different ratios (Ratio 1 and Ratio2). b) Example of state map dynamics in Fp2 channel during the discrimination and response periods. The arrow tail indicates the ratios coordinates (Ratio 1 in abscissa axis and Ratio2 in ordinate axis) during the discrimination (Dis) period, and arrow tip indicates the coordinates of ratios during the response period (Resp). An overall displacement towards higher ratio1 and ratio 2 is observed for this channel. c) Network of electrodes with significant differences for ratio 1 (Blue), ratio 2 (yellow), or both (green). d) Comparison of performance and neural activity during the discrimination period. Red and yellow indicate positive correlations, while blue indicates negative correlations. Asterisks indicate significant Spearman correlations. On the left panel, Rs (Spearman's Rho) correlation values between ratio 1 and performance are presented. Ratio 1 was significantly correlated to tactile width performance in electrodes positioned in frontal (Fp2), parietal (P3 and P4), temporal (T8), and occipital regions (02). On the right panel the Spearman correlation values between ratio 2 and performance are presented. Ratio 2 was correlated to tactile performance in P3 and P4 electrodes. e) Example of individual values of ratio 1 and performance for electrodes Fp2, P3 and P4. f) Example of individual values of ratio 2 and performance for electrodes P3 and P4. $\mathrm{g}-\mathrm{h}$ ) The same data as in panels e$f$ is presented, but only for one channel. The red circles show ratio 1 values in channel Fp2 (panel e) and for channel P3 (panel h) for three additional subjects tested in an easier version of the task. For panels $\mathrm{g}$ and $\mathrm{h}$ (after statistical analysis), data points that were superimposed in the graph, were increased by 0.2 units in the $\mathrm{X}$ axis to facilitate visualization. 

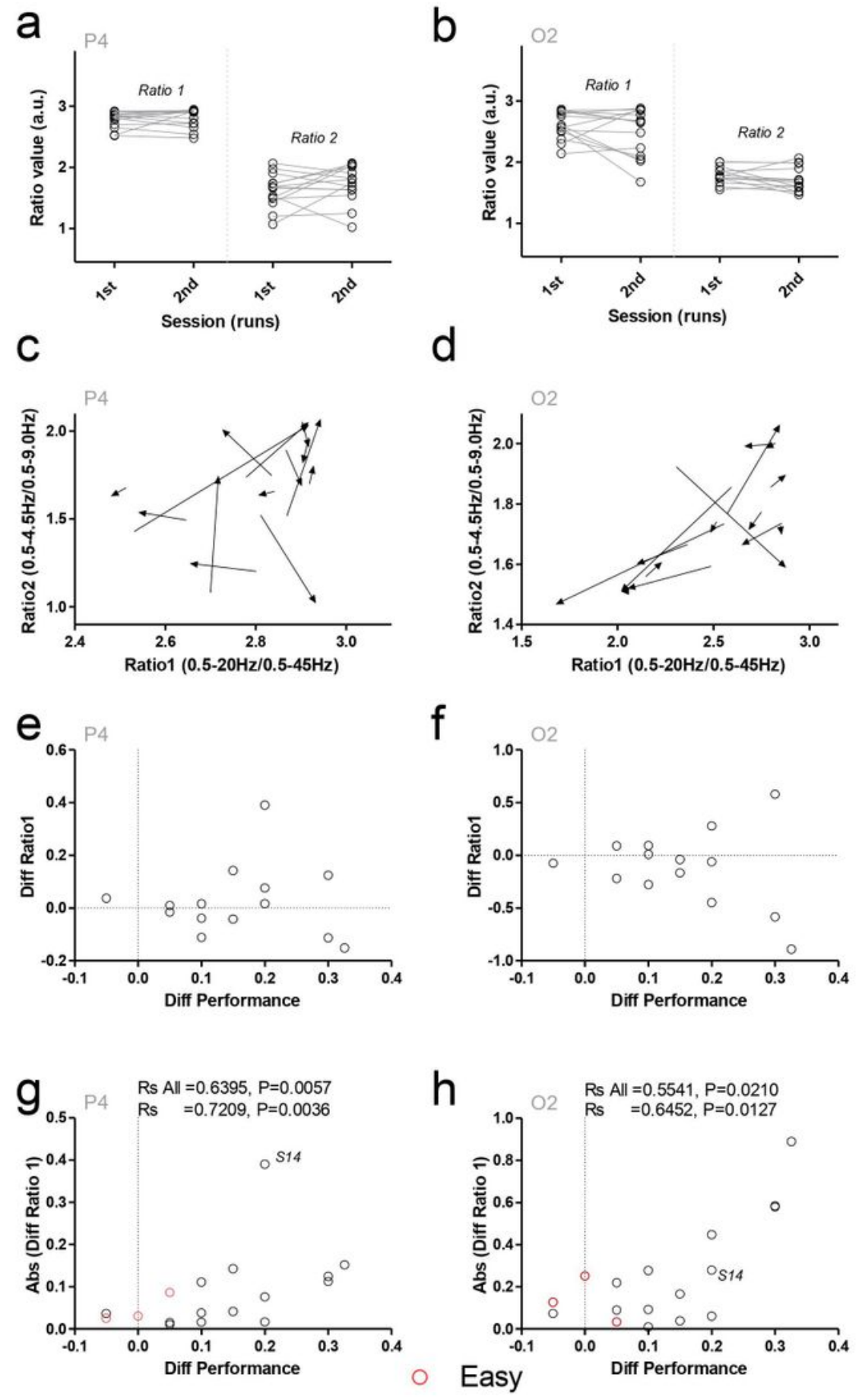

\section{Figure 3}

Ratio 1 dynamics reflect the changes in performances within subjects. $a$ and b) No clear differences between ratio 1 or ratio 2 were observed between the first and second run. $c$ and d) Simultaneous analysis of ratio 1 and ratio 2 suggested an overall pattern of change, especially in ratio 2, associated with the lower right qudrant of the panel. e and $f$ ) vector displacement, reflecting the changes in performance and ratio 1 occurring between the first and the second run, presented an overall symmetrical 
pattern around the value of 0 for the $Y$ axis. $g$ and $h$ ) calculating the absolute value of difference (Diff) for channels P4 (g) and $02(\mathrm{~h})$ allowed predicting changes in performance between the first and the second run.

\section{Supplementary Files}

This is a list of supplementary files associated with this preprint. Click to download.

- T1.jpg

- T2.jpg

- T3.jpg 